\title{
The Comparison and Validity of Troponin I Assay Systems in Diagnosing Myocardial Ischemic Injury After Surgical Coronary Revascularization
}

\author{
C. Bruce Martin, MBBS, FRCA,* Andrew D. Shaw, MBBS, FRCA,* Janos Gal, MD, PhD,* \\ Natarajan Aravindan, PhD,* Frank Murphy, MPath, $\dagger$ David Royston, MBBS, FRCA, \\ and Bernhard J. Riedel, MBChB, FCA, MMed, FAHA*
}

Objective: A prospective observational study was conducted to test the agreement between 2 commercially available automated cardiac troponin-I immunoassay systems (Opus Plus, Behring Diagnostics UK Ltd, Hounslow, UK; AxSYM, Abbott Laboratories, Abbott Park, IL) and to determine a normal reference range and threshold value indicative of perioperative myocardial infarction (PMI) after elective coronary artery bypass graft (CABG) surgery for the Opus Plus system.

Design: Prospective, observational study.

Setting: Single institution, cardiothoracic specialty hospital.

Participants: Seventy patients undergoing elective CABG surgery.

Interventions: After institutional review board approval, patients received standardized anesthetic, surgical, and myocardial preservation techniques. Serial electrocardiographs, creatine kinase-MB, troponin-I, and perioperative outcome data were collected. Correlation between the immunoassay systems was tested using 124 duplicate samples from the first 18 patients. The normal reference range and threshold value indicative of PMI were tested for the Opus Plus system using duplicate samples from all 70 patients.

D ESPITE ADVANCES IN myocardial protective strategies, ischemic injury often complicates coronary artery bypass graft $(\mathrm{CABG})$ surgery. Accurate diagnosis and timely intervention of perioperative myocardial infarction (PMI) are vital because major ischemic injury is associated with reduced hospital $^{1-4}$ and long-term ${ }^{5,6}$ survival. Similarly, more recent data suggest that minor ischemic injury after CABG surgery is also associated with adverse short-term ${ }^{7,8}$ and medium-term ${ }^{9}$ outcome. Accurate assessment of ischemic injury will facilitate the comparison and optimization of myocardial protective strategies with further improved surgical outcome.

The Joint European Society of Cardiology/American College of Cardiology Committee for redefinition of myocardial infarction (MI) currently considers cardiac troponin isozyme (cTn) as the biomarker of choice for myocardial injury. ${ }^{10}$ This is on account of the improved sensitivity and specificity of cTn for acute MI, both nonsurgical ${ }^{11,12}$ and perioperative $e^{7,13-17}$ and for reperfusion injury after thrombolysis. ${ }^{18}$ The consensus document, however, fails to set standard criteria for PMI after cardiac surgery.

From the Departments of *Anesthesiology and Critical Care and $\dagger$ Biochemistry, Royal Brompton and Harefield NHS Trust, London, United Kingdom.

Address reprint requests to Bernhard J. Riedel, MBChB, FCA, MMed, FAHA, Division of Anesthesiology and Critical Care, Unit 42, The University of Texas M.D. Anderson Cancer Center, 1515 Holcombe Boulevard, Houston, TX, 77030. E-mail: briedel@ mdanderson.org

(C) 2005 Elsevier Inc. All rights reserved.

1053-0770/05/1903-0003\$30.00/0

doi:10.1053/j.jvca.2005.03.003
Measurements and Main Results: Peak troponin-I concentrations (median [interquartile range]) differed significantly when measured by the Opus Plus and AxSYM immunoassay systems (5.61 [3.20-22.35] $\mu \mathrm{g} / \mathrm{L} v \mathbf{4 6 . 5 0}$ [14.55-70.95] $\mu \mathrm{g} / \mathrm{L}$, respectively; $p<0.001$ ). There was clear proportional bias that was corrected with log transformation of the raw data. By using confidence interval and receiver operating characteristic curve analysis, the authors showed that a value $\geq 15$ $\mu \mathrm{g} / \mathrm{L}$ was indicative of PMI (Opus Plus system) and accordingly report a $35.7 \%$ (2.9\% Q-wave) overall incidence of PMI in this study population $(n=70)$.

Conclusions: These data highlight differences between commercially available troponin-I assay systems. The authors recommend that each institution establish a local reference range and threshold indicative of perioperative myocardial infarction for its specific patient population and assay system and provide sample methodology.

(c) 2005 Elsevier Inc. All rights reserved.

KEY WORDS: coronary artery bypass graft surgery, perioperative myocardial infarction, troponin-I, receiver operating characteristic curve analysis

After the introduction of a commercially available automated troponin-I (cTn-I) immunoassay system into the authors' clinical laboratory and based on the lack of standardization between such systems, a prospective observational study was conducted to (1) test the hypothesis that there would be no difference between 2 commercially available cTn-I immunoassay systems (Opus Plus analyser, Behring Diagnostics UK Ltd, Hounslow, UK; AxSYM Immunoassay system, Abbott Laboratories, Abbot Park, IL), and (2) establish a normal reference range for the cTn-I system (Opus Plus) implemented at the authors' institution. This reference range would be representative of patients who did not suffer a PMI after elective CABG surgery, with a value above the upper limit of this reference range being indicative of troponin release consequent to ischemic injury rather than surgical manipulation.

\section{METHODS}

After institutional review board approval, informed consent was obtained from 70 consecutive patients scheduled for elective CABG surgery. Inclusion criteria consisted of significant stenosis in at least 2 coronary arteries with preserved left ventricular systolic function. Exclusion criteria were acute preoperative MI (within 7 days of surgery), preexisting conduction or rhythm abnormalities (such as left bundlebranch block, atrial fibrillation), and revision surgery or the need for a combined surgical procedure (such as CABG and carotid endarterectomy or valvular surgery).

All previously prescribed medications were continued preoperatively. Anesthesia was induced with propofol $(1-2 \mathrm{mg} / \mathrm{kg})$, fentanyl $(8-10 \mu \mathrm{g} / \mathrm{kg})$, and pancuronium bromide $(0.15 \mathrm{mg} / \mathrm{kg})$ used for muscle relaxation. Before, during, and after nonpulsatile cardiopulmonary bypass $(\mathrm{CPB})$, anesthesia was maintained with isoflurane (0.5-1 minimum alveolar concentration) and a propofol infusion $(3 \mathrm{mg} / \mathrm{kg} / \mathrm{h})$. Propofol was continued in the intensive care unit (ICU) until patients met 
Fig 1. Bland-Altman plot of assay comparison. Data shows clear proportional bias. Opus Plus and AxSYM on the $x$ and $y$ axis represent $c T n$-I concentrations measured by these assay systems, respectively.

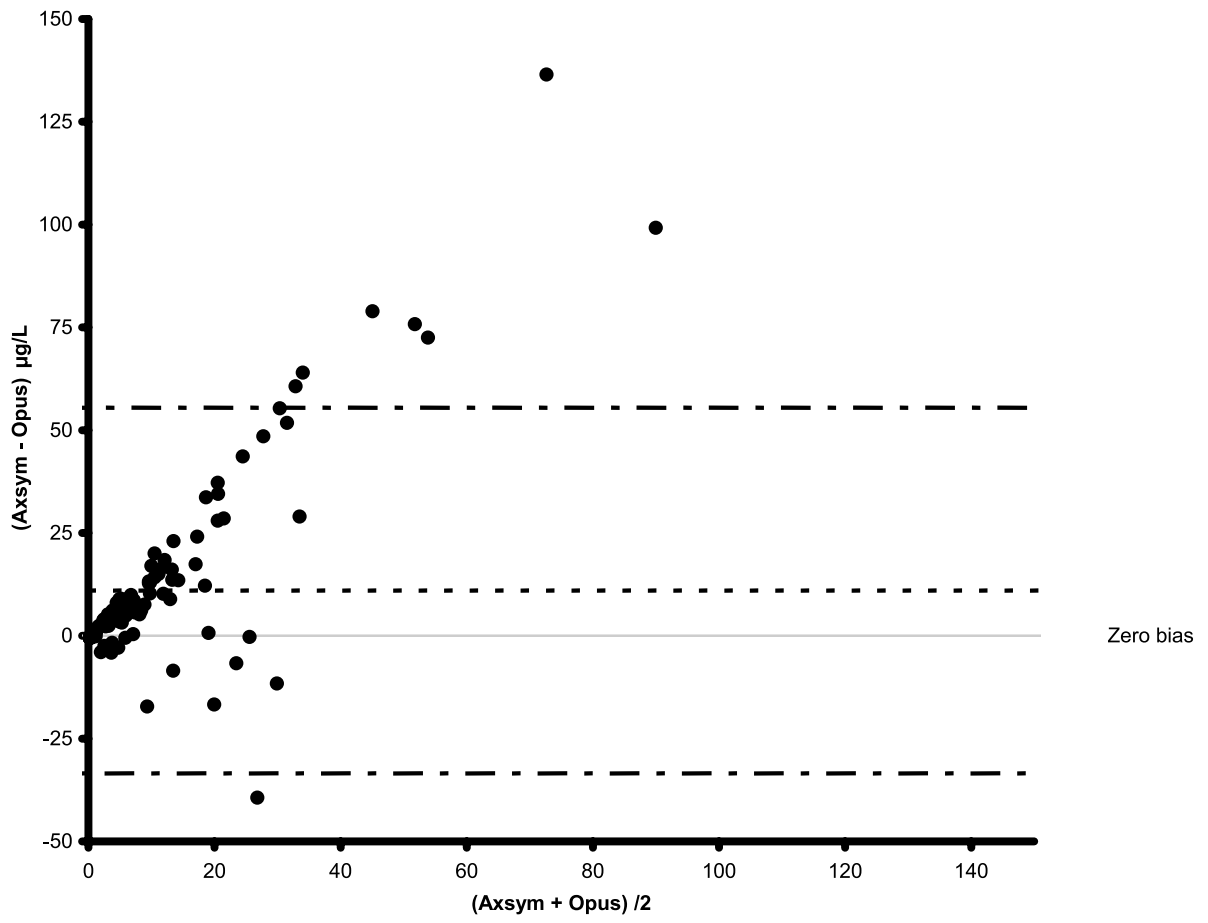

To investigate the extent of agreement between different cTnI assays, the Opus Plus and the AxSYM methods were compared using 124 duplicate samples from the first 18 patients. Thereafter, serum samples from all 70 patients were analyzed using the Opus Plus method to determine a local reference range and thus establish a threshold indicative of PMI after elective CABG surgery for the authors' institution.

The Opus Plus method uses a fluorogenic enzyme-linked, 2-site (sandwich) immunoassay technique with goat polyclonal antibodies to troponin. The AxSYM method uses a microparticle enzyme immunoassay technique with mouse monoclonal antibodies to the cardiac isoform of troponin I. Intra-assay and interassay variability is 5.0\% and 5.3\% and $4.4 \%$ and $6.3 \%$ for the Opus Plus and AxSYM methods, respectively. For both assays, $<0.5 \mu \mathrm{g} / \mathrm{L}$ is given as the normal reference range for apparently healthy individuals and $2.0 \mu \mathrm{g} / \mathrm{L}$ the diagnostic cutoff for acute MI in the nonsurgical population.

Serial 12-lead ECGs were recorded preoperatively and again on postoperative days 1,3, and 5. Two independent physicians, blinded to the laboratory data, interpreted the electrocardiograms. If a consensus could not be reached, the opinion of a third physician was obtained.

PMI was diagnosed when the following ECG and CK-MB criteria were met:

1. ECG changes according to the Minnesota code criteria ${ }^{19}$

a. Q-wave PMI: the appearance of new and persistent Q-waves $>0.04$ seconds in duration in at least 2 contiguous leads or equivalent $\mathrm{R}$-wave increments leading to an $\mathrm{R} / \mathrm{S}$ ratio of $>1$ in leads V1 and V2 of the standard 12-lead ECG.

b. Non-Q-wave PMI: the appearance of new and persistent $(>24$ hours) conduction abnormalities or ST-T alterations (ST-segment depression or elevation $>0.1 \mathrm{mV}$ at 0.08 seconds after the $\mathrm{J}$-point or T-wave inversion) in at least 2 contiguous leads of the standard 12-lead ECG.

2. CK-MB activity $>50 \mathrm{IU} / \mathrm{L}$ lasting for more than 12 hours ${ }^{20-22}$ or occurring after 21 hours of reperfusion. ${ }^{23}$

When only one ECG or CK-MB criterion was met, PMI status was regarded as "undefined."

Data relating to the need for cardiovascular support, incidence of allowed to coagulate, and then centrifuged at $3,500 \mathrm{rpm}$ for 15 minutes at $4^{\circ} \mathrm{C}$. Serum was then stored at $-0^{\circ} \mathrm{C}$ until assays were performed. 


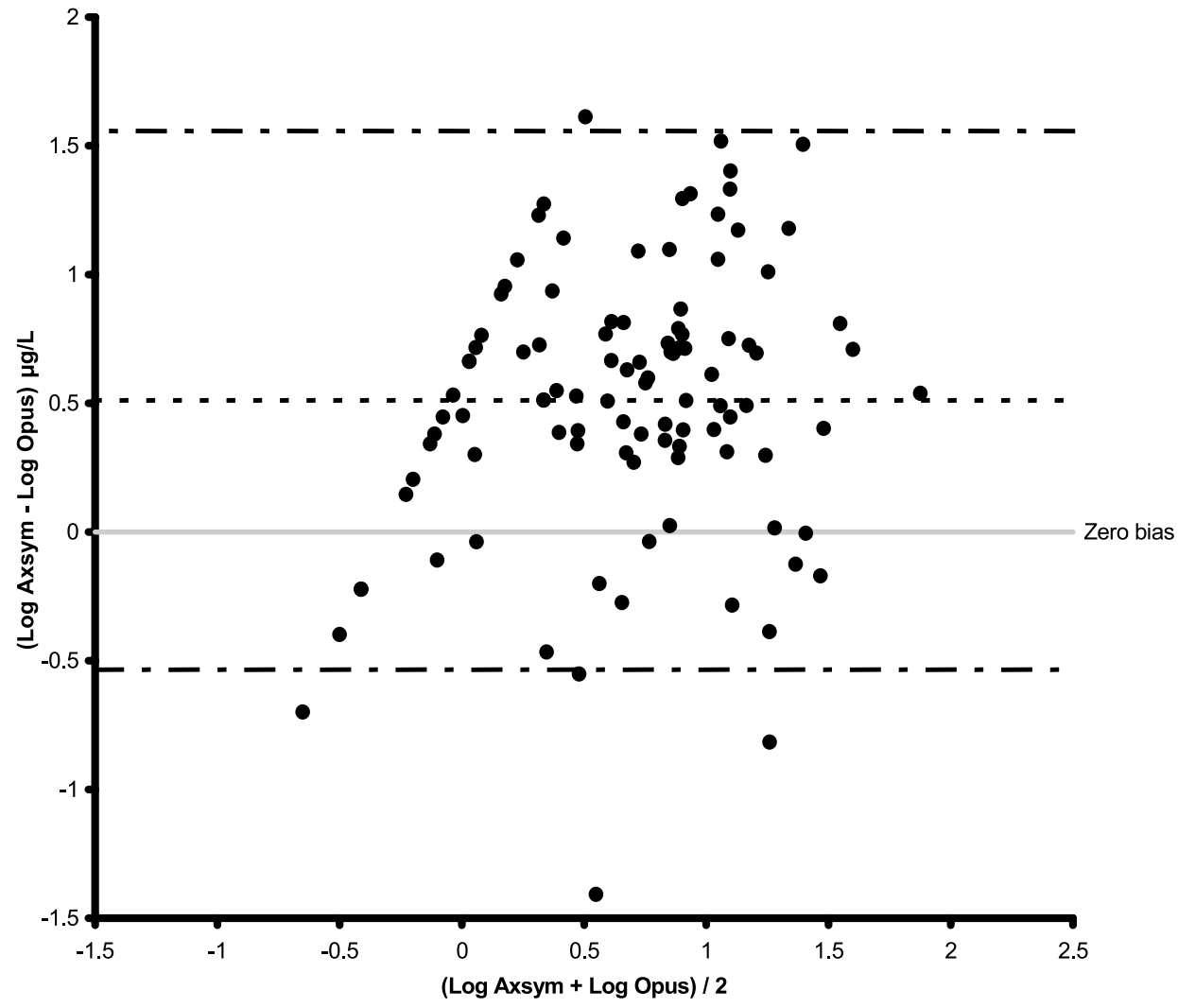

Fig 2. Bland-Altman plot of assay comparison with correction of bias by log-transformation. Opus Plus and AxSYM on the $x$ and $y$ axis represent $c T n-I$ concentrations measured by these assay systems, respectively. postoperative atrial fibrillation (requiring treatment), duration of mechanical ventilation, ICU length of stay, and hospital length of stay were recorded. Cardiovascular support was defined as the requirement for inotropic support (dopamine, $>3 \mu \mathrm{g} / \mathrm{kg} / \mathrm{min}$, or epinephrine infusion), pacing, or antiarrhythmic therapy for malignant arrhythmias.

Data are presented as mean \pm standard error of the mean or as a percentage of the study group unless stated otherwise. To evaluate agreement between the 2 commercially available cTn-I assays, data were analyzed using the method of Bland and Altman. ${ }^{24,25}$ Parametric and nonparametric data were analyzed using the appropriate test (eg, unpaired $t$ test for continuous parametric variables, Fisher exact test for categoric variables, and Mann-Whitney $U$ test for nonparametric variables.) One-way analysis of variance or Kruskal-Wallis tests were performed to assess the statistical significance of observed differences between multiple groups. Receiver operating characteristic (ROC) curves were plotted to assess the utility of troponin measurement for diagnosis of PMI. In all cases, a $p$ value of $<0.05$ was regarded as statistically significant. Analyses were performed using GraphPad Prism version 3.00 (GraphPad Software Inc, San Diego, CA) and Excel 2000 (Microsoft Inc, Redmond, WA) with the Analyse-It add-in (Analyse-It Ltd, Leeds, UK).

\section{RESULTS}

All patients had normal preoperative CK-MB $(<24$ IU/L) and $\mathrm{cTn}-\mathrm{I}(<2 \mu \mathrm{g} / \mathrm{L})$ values. In addition, preoperative cTn-I concentrations did not differ significantly between groups.

One hundred twenty-four duplicate samples were analyzed from the first 18 patients. Peak cTn-I protein concentrations (median [interquartile range]) differed significantly (5.61 [3.2022.35] $\mu \mathrm{g} / \mathrm{L} v 46.50$ [14.55-70.95] $\mu \mathrm{g} / \mathrm{L} ; p<0.001)$ when measured using the Opus Plus and AxSYM assay systems, respectively. Although good correlation $\left(R^{2}=0.7\right)$ was observed between the 2 assay systems, the Bland-Altman plot of the data revealed clear proportional bias (Fig 1) that corrected on $\log$ transformation of the raw data (Fig 2). According to the Opus Plus assay, 5 of 18 patients had peak cTn-I values suggestive of PMI ( $>15 \mu \mathrm{g} / \mathrm{L}$, see later). However, 3 of these patients had values below the median peak cTn-I value (46.5 $\mu \mathrm{g} / \mathrm{L}$ ) for the AxSYM assay. Furthermore, 2 patients with peak cTn-I values $<15 \mu \mathrm{g} / \mathrm{L}$ by the Opus Plus method had exceptionally high $(>80 \mu \mathrm{g} / \mathrm{L})$ peak cTn-I values when measured by the AxSYM method.

After CABG surgery, the 70 patients were divided into 1 of 3 groups according to whether or not they met the predefined criteria for PMI based on CK-MB and ECG changes (see Methods). The groups consisted of non-PMI ( $\mathrm{n}=40,57.1 \%)$, PMI $(\mathrm{n}=9,12.9 \%)$, and undefined $(\mathrm{n}=21,30.0 \%)$. Of the 9 patients with PMI, 2 (2.9\%) patients suffered a Q-wave PMI, and $7(10.0 \%)$ patients suffered a non-Q-wave PMI.

Demographic and intraoperative data for the 3 patient groups (non-PMI, undefined, and PMI) are summarized in Table 1. Groups were similar in terms of the sex ratio, age, weight, preoperative comorbid risk factors, medications, angina score, preoperative ventricular function, and number of stenosed coronary arteries. Intraoperative data, including number of coronary arteries grafted and duration of CPB and aortic crossclamping, were also similar between groups. All patients had a good clinical outcome with no incidence of malignant arrhythmia, cardiac failure, or need for intra-aortic balloon counterpulsation. There were no perioperative deaths, and all patients 
Table 1. Demographic Data of Non-PMI, Undefined, and PMI Groups

\begin{tabular}{|c|c|c|c|c|}
\hline & Non-PMI & Undefined & PMI & $p$ Value \\
\hline Patients, no. (\%) & $40(57.1)$ & $21(30.0)$ & $9(12.9)$ & \\
\hline Sex ratio & $92.5 \%$ male & $90.5 \%$ male & $100 \%$ male & NS (0.62) \\
\hline Weight $(\mathrm{kg})$ & $80.0 \pm 2.0$ & $78.6 \pm 2.3$ & $79.7 \pm 3.6$ & NS $(0.90)$ \\
\hline \multicolumn{5}{|c|}{ Preoperative comorbidities (\%) } \\
\hline Hypertension & 35.0 & 38.1 & 22.2 & NS $(0.70)$ \\
\hline Hypercholesterolemia & 67.6 & 68.4 & 88.9 & NS (0.44) \\
\hline \multicolumn{5}{|l|}{ Preoperative therapy $(\%)$} \\
\hline Aspirin & 70.0 & 80.9 & 88.9 & NS (0.39) \\
\hline $\mathrm{Ca}^{2+}$ antagonists & 50.0 & 38.1 & 66.7 & NS (0.63) \\
\hline Nitrates & 45.0 & 42.9 & 66.7 & NS (0.45) \\
\hline ACE inhibitors & 12.5 & 14.3 & 0 & NS $(0.50)$ \\
\hline Diuretics & 7.5 & 9.5 & 33.3 & NS (0.08) \\
\hline Angina (CCSC) & & & & NS (0.96) \\
\hline I-II & 80.0 & 66.7 & 55.6 & \\
\hline III-IV & 20.0 & 33.3 & 44.4 & \\
\hline Previous MI & 62.5 & 47.6 & 44.4 & NS (0.41) \\
\hline \multicolumn{5}{|l|}{ Angiography } \\
\hline SWMA & 26.9 & 28.6 & 22.2 & NS (0.72) \\
\hline LVEF $>50 \%$ & 100.0 & 100.0 & 100.0 & NS (1.00) \\
\hline LVEDP $>12 \mathrm{mmHg}$ & 20.0 & 9.5 & 22.2 & NS (0.53) \\
\hline No. diseased vessels & $2.7 \pm 0.1$ & $2.7 \pm 0.1$ & $2.8 \pm 0.2$ & NS (0.92) \\
\hline \multicolumn{5}{|l|}{ Operative data } \\
\hline No. of grafts & $2.8 \pm 0.1$ & $2.7 \pm 0.1$ & $2.8 \pm 0.2$ & NS $(0.91)$ \\
\hline CPB duration (min) & $72.4 \pm 3.5$ & $75.0 \pm 5.7$ & $85.7 \pm 6.4$ & NS $(0.30)$ \\
\hline AXC duration (min) & $36.1 \pm 3.2$ & $38.4 \pm 3.4$ & $48.6 \pm 4.2$ & NS (0.18) \\
\hline
\end{tabular}

Abbreviations: NIDDM, noninsulin-dependent diabetes mellitus; NS, not significant; ACE, angiotensin-converting enzyme; LVEF, left ventric ular ejection fraction; LVEDP, left ventricular end-diastolic pressure; SWMA, segmental wall motion abnormality; AXC, aortic cross-clamp.

survived to hospital discharge. Peak cTn-I concentration and time to peak cTn-I concentration according to the Opus Plus method for the 3 groups are presented in Table 2.

Assuming a normal/Gaussian distribution for most clinical assay data, the upper limit of "normal" is defined as the mean value plus 1.96 SD (ie, at the 97.5th percentile). However, it has recently been suggested that a value of mean plus $3 \mathrm{SD}$ (99.5th percentile) defines this upper limit of a "normal" population more accurately. ${ }^{26}$ Using these definitions, in the authors' laboratory the upper reference limit for "normal" peak cTn-I protein concentration after uncomplicated on-pump coronary revascularization is $15.7 \mu \mathrm{g} / \mathrm{L}$ (97.5th percentile) and 20 $\mu \mathrm{g} / \mathrm{L}$ (99.5th percentile), respectively, as reflected by the nonPMI group. Accordingly, values below or above the upper reference limit would most likely be representative of surgical trauma or ischemic myocardial injury, respectively.

Because, in this case, there is no meaning for a lower limit of normal, the ROC curve method is suitable as a means of

Table 2. Peak cTn-I Concentrations for Non-PMI and P-MI Groups

\begin{tabular}{lccc}
\hline & $\begin{array}{c}\text { No. of Patients } \\
(\%)\end{array}$ & Peak cTn-I & $\begin{array}{c}\text { Hours After } \\
\text { reperfusion }\end{array}$ \\
\hline Non-PMI & $40(57.1)$ & $7.1 \pm 4.3 \mu \mathrm{g} / \mathrm{L}$ & $8.6 \pm 5.6$ \\
Non-Q-wave PMI & $7(10.0)$ & $27.2 \pm 13.3 \mu \mathrm{g} / \mathrm{L}$ & $12.9 \pm 8.1$ \\
Q-wave PMI & $2(2.9)$ & $67.3 \pm 36.4 \mu \mathrm{g} / \mathrm{L}$ & $33.0 \pm 21.2$ \\
\hline
\end{tabular}

verifying the reference range calculated from confidence intervals above. The cTn-I data from the non-PMI and PMI patient groups were plotted to generate an ROC curve for the ability of

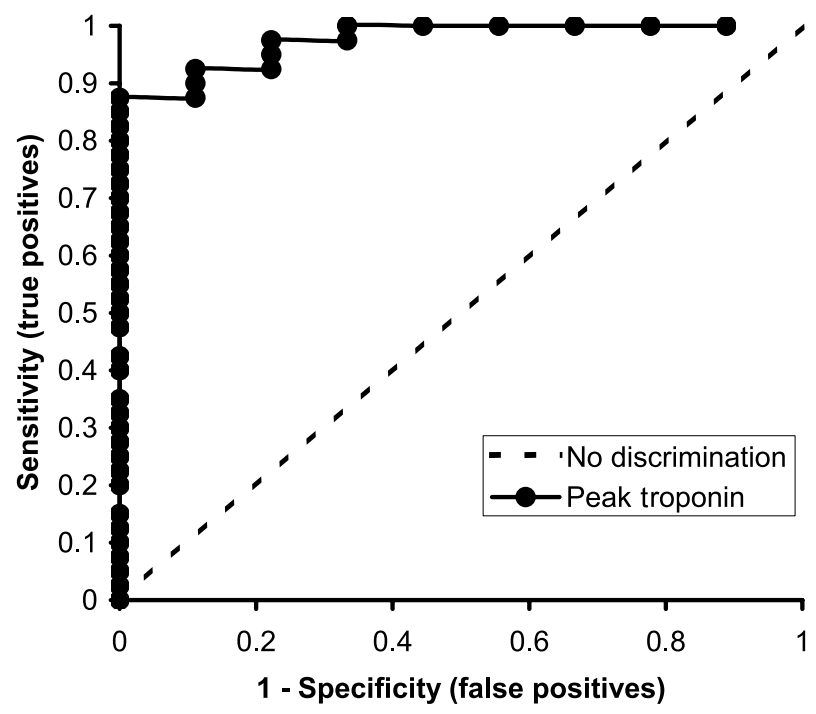

Fig 3. ROC curve showing utility of $\mathrm{cTn}$-I for the diagnosis of PMI after elective CABG surgery. 
Table 3. Perioperative Complications and Duration of Postoperative Care

\begin{tabular}{lcccc}
\hline & Non-PMI & PMI & $p$ Value & Relative Ratio (95\% Cl) \\
\hline Inotropic support (\%) & 6.6 & 28 & 0.03 & $4.2(1.2-14.8)$ \\
Rhythm disturbance (\%) & 32 & 76 & 0.58 & 0.10 \\
Ventilation (h) & $6.6 \pm 0.9$ & $5.0 \pm 0.5$ & 0.94 & $0.6-2.8)$ \\
ICU-LOS (h) & $20.4 \pm 4.7$ & $20.0 \pm 2.0$ & 0.76 \\
Hospital LOS (d) & $6.3 \pm 0.4$ & $6.4 \pm 0.3$ & \\
\hline
\end{tabular}

Abbreviations: $\mathrm{Cl}$, confidence interval; LOS, length of stay.

cTn-I to detect PMI (Fig 3). Using the upper-left corner of the curve, a value of $15 \mu \mathrm{g} / \mathrm{L}$ was defined as the cut point for PMI. This value had a sensitivity (true-positive rate) of $92.5 \%$ and a specificity (true-negative rate) of $88.9 \%$. An area under the ROC curve of 0.975 represents excellent assay utility.

Using $15 \mu \mathrm{g} / \mathrm{L}$ as the cut point value, as suggested by both the ROC curve and the confidence interval (97.5th percentile) methods, the authors reclassified the 21 patients in the "undefined" group into either the non-PMI (peak cTn-I $<15 \mu \mathrm{g} / \mathrm{L}$ ) or PMI (peak cTn-I $\geq 15 \mu \mathrm{g} / \mathrm{L}$ ) groups. This resulted in a diagnosis of PMI in 25 of the 70 patients $(35.7 \%)$ in the present study population.

When PMI was defined using the cutpoint $\geq 15 \mu \mathrm{g} / \mathrm{L}$ for cTn-I, the authors noted that ECG changes were inconsistent, with $36.0 \%(9 / 25)$ false negative (according to cTn-I criteria failed to show new postoperative ECG changes indicative of PMI) and $17.8 \%$ (8/45) false-positive incidence of PMI after surgery. CK-MB levels showed better diagnostic utility than ECG, with $8.0 \%$ (2/25) false-negative and $15.6 \%$ (7/45) falsepositive incidence of PMI after surgery.

The requirement for cardiovascular support and the incidence of postoperative complications are summarized in Table 3. Requirement for inotropic support was significantly increased in the PMI group. Although more patients in the PMI group experienced arrhythmia, this did not reach statistical significance.

\section{DISCUSSION}

The aim of this study was 2-fold: (1) to compare the correlation between 2 commercially available cTn-I assay systems and (2) to identify a value for cTn-I above which the authors would be fairly certain that a patient had sustained an ischemic myocardial injury after CABG surgery. In keeping with data by Kao et $a l,{ }^{27}$ the authors report a significant difference between the AxSYM and Opus Plus assay systems. By using certain characteristics of the Gaussian distribution and then confirming findings with a second, independent methodology (ROC curve analysis), a cut point ( $>15 \mu \mathrm{g} / \mathrm{L}$, Opus Plus) was established that is indicative of ischemic myocardial injury after CABG surgery.

The role of cTn-I for the diagnosis of $\mathrm{PMI}^{7,8,10,14-17}$ is now well established; however, the fact that the authors and others report different values between commercially available cTn-I assay systems and the fact that the upper reference values differ significantly between various studies ${ }^{7,15,17}$ serves to further highlight the need for individual centers to determine reference ranges suitable to their clinical environment; that is, until such time as cTn-I assay systems, surgical techniques (on-pump $v$ off-pump OPCAB), and cardioprotective strategies are standardized.

The authors noted a clear proportional bias between the 2 assay systems used in this study. This is of concern when a high value for a test is presumably indicative of increasing severity of pathology. If a laboratory changes its assay without warning then there is the possibility for over- or, even worse, underdiagnosis of PMI, especially because the adverse relationship that exists between PMI and patient outcome ${ }^{1-9,17}$ is increasingly recognized and may warrant early intervention. Accordingly, an overall PMI rate of $35.7 \%$ in this series of patients was reported. The authors believe that this is a more accurate estimate of perioperative myocardial injury than previously published estimates ${ }^{3,4,6}$ because it includes all grades of ischemic injury rather than just transmural MIs. The authors found that a cTn-I value of $\geq 15 \mu \mathrm{g} / \mathrm{L}$ had a sensitivity (true-positive rate) of $92.5 \%$ and a specificity (true-negative rate) of $88.9 \%$ for the diagnosis of PMI, which the authors believe are acceptable parameters for use in routine clinical practice. The area under the ROC curve of 0.975 supports this premise. Although this is not a study of cardioplegia methods, these data suggest cardioprotective strategies may be improved.

A major limitation of this study is the lack of a true diagnostic gold standard to compare with the troponin measurement because ECG changes and CK-MB levels are inaccurate in this setting. This is supported by Van Velde et $\mathrm{al}^{28}$ who showed poor concordance between changes in enzyme activity $(\mathrm{CK}$, CK-MB, aspartate aminotransferase, and the lactate dehydrogenase-1/lactate dehydrogenase-2 ratio) and ultimate evidence of PMI at autopsy after cardiac surgery. In the present series, ECG changes alone would have led to an erroneous diagnosis regarding PMI more than half of the time.

It is expected that biomarker release will occur in all patients during cardiac surgery (related to both ischemic and surgical injury) and therefore an attempt to differentiate procedural injury from true ischemic injury by defining biomarker release in the reference control group (those without PMI according to CK-MB and ECG criteria) may have been facilitated by the use of echocardiographic or other imaging criteria.

Other limitations to this study include the fact that the predefined criteria for PMI differ from those published in the consensus document of the Joint European Society of Cardiology/American College of Cardiology Committee for redefinition of myocardial infarction. ${ }^{10}$ That said, the comprehensive guidelines within the consensus document focus predominantly on the nonsurgical setting and the criteria for ECG and CK-MB changes, as supported by the cardiac 
surgery literature, differ only slightly from those proposed. Examples of minor differences include the following: a Q-wave MI was defined as a Q-wave duration $>0.04$ seconds, whereas the consensus document proposes a $\mathrm{Q}$-wave duration $>0.03$ seconds. CK-MB levels significant for PMI were set at twice the upper reference limit, similar to that proposed for total CK by the consensus document, whereas the consensus document recommends that significant CK-MB values are those that exceed the 99th percentile of CK-MB values in a reference control group.

Finally, because the patient population was relatively healthy, with normal ejection fractions, and few postoperative morbid events, this study is underpowered from a clinical outcome point of view. Nevertheless, having established a reference range for cTn-I elevation after CABG surgery for the authors' institution, the authors now have the ability to correlate a possible relationship between severity of elevated cTn-I with timing of elevation (Table 2) and adverse clinical outcome in a future adequately powered study. Furthermore, the authors provide a description of a method, which is simple to reproduce using tests that are already available and which are self-confirmatory, so busy units may verify for themselves that their laboratory and clinical impressions are accurate. It does not require large numbers of patients or tests per patient and thus is affordable. This may allow centers to establish a local baseline incidence of myocardial injury after elective CABG surgery, against which newer myocardial protective strategies can be compared.

\section{REFERENCES}

1. Gray RJ, Matloff JM, Conklin CM, et al: Perioperative myocardial infarction: Late clinical course after coronary artery bypass surgery. Circulation 66:1185-1189, 1982

2. Namay DL, Hammermeister KE, Zia MS, et al: Effect of perioperative myocardial infarction on late survival in patients undergoing coronary artery bypass surgery. Circulation 65:1066-1071, 1982

3. Chaitman BR, Alderman EL, Sheffield LT, et al: Use of survival analysis to determine the clinical significance of new Q waves after coronary bypass surgery. Circulation 67:302-309, 1983

4. Slogoff S, Keats AS: Does perioperative myocardial ischemia lead to postoperative myocardial infarction? Anesthesiology 62:107114, 1985

5. Force T, Hibberd P, Weeks G, et al: Perioperative myocardial infarction after coronary artery bypass surgery. Clinical significance and approach to risk stratification. Circulation 82:903-912, 1990

6. Guiteras Val P, Pelletier LC, Hernandez MG, et al: Diagnostic criteria and prognosis of perioperative myocardial infarction following coronary bypass. J Thorac Cardiovasc Surg 86:878-886, 1983

7. Greenson N, Macoviak J, Krishnaswamy P, et al: Usefulness of cardiac troponin I in patients undergoing open heart surgery. Am Heart J 141:447-455, 2001

8. Dehoux M, Provenchere S, Benessiano J, et al: Utility of cardiac troponin measurement after cardiac surgery. Clin Chim Acta 311:4144, 2001

9. Klatte K, Chaitman BR, Theroux P, et al: Increased mortality after coronary artery bypass graft surgery is associated with increased levels of postoperative creatine kinase-myocardial band isoenzyme release: Results from the GUARDIAN trial. J Am Coll Cardiol 38: 1070-1077, 2001

10. Alpert JS, Thygesen K, Antman E, et al: Myocardial infarction redefined-A consensus document of The Joint European Society of Cardiology/American College of Cardiology Committee for the redefinition of myocardial infarction. J Am Coll Cardiol 36:959-969, 2000

11. Mair J, Artner-Dworzak E, Lechleitner P, et al: Cardiac troponin $\mathrm{T}$ in diagnosis of acute myocardial infarction. Clin Chem 37:845-852, 1991

12. Mair J: Markers for early diagnosis of myocardial infarction. Lancet 342:1553-1554, 1993

13. Mair J, Wieser C, Seibt I, et al: Troponin $\mathrm{T}$ to diagnose myocardial infarction in bypass surgery. Lancet 337:434-435, 1991

14. Mair P, Mair J, Seibt I, et al: Cardiac troponin T: A new marker of myocardial tissue damage in bypass surgery. J Cardiothorac Vasc Anesth 7:674-678, 1993
15. Mair J, Larue C, Mair P, et al: Use of cardiac troponin I to diagnose perioperative myocardial infarction in coronary artery bypass grafting. Clin Chem 40:2066-2070, 1994

16. Etievent JP, Chocron S, Toubin G, et al: Use of cardiac troponin I as a marker of perioperative myocardial ischemia. Ann Thorac Surg 59:1192-1194, 1995

17. Benoit MO, Paris M, Silleran J, et al: Cardiac troponin I: its contribution to the diagnosis of perioperative myocardial infarction and various complications of cardiac surgery. Crit Care Med 29:1880-1886, 2001

18. Apple FS, Voss E, Lund L, et al: Cardiac troponin, CK-MB and myoglobin for the early detection of acute myocardial infarction and monitoring of reperfusion following thrombolytic therapy. Clin Chim Acta 237:59-66, 1995

19. Blackburn H, Keys A, Simonson E, et al: The electrocardiogram in population studies. A classification system. Circulation 21:11601175,1960

20. Delva E, Maille JG, Solymoss BC, et al: Evaluation of myocardial damage during coronary artery grafting with serial determinations of serum CPK MB isoenzyme. J Thorac Cardiovasc Surg 75:467-475, 1978

21. Lee TH, Goldman L: Serum enzyme assays in the diagnosis of acute myocardial infarction. Recommendations based on a quantitative analysis. Ann Intern Med 105:221-233, 1986

22. Graeber GM, Shawl FA, Head HD, et al: Changes in serum creatine kinase and lactate dehydrogenase caused by acute perioperative myocardial infarction and by transatrial cardiac surgical procedures. J Thorac Cardiovasc Surg 92:63-72, 1986

23. Farah SY, Moss DW, Ribeiro P, et al: Interpretation of changes in the activity of creatine kinase $\mathrm{MB}$ isoenzyme in serum after coronary artery bypass grafting. Clin Chim Acta 141:219-225, 1984

24. Bland JM, Altman DG: Statistical methods for assessing agreement between two methods of clinical measurement. Lancet 1:307-310, 1986

25. Bland JM, Altman DG: Comparing methods of measurement: Why plotting difference against standard method is misleading. Lancet 346:1085-1087, 1995

26. Jaffe AS, Ravkilde J, Roberts R, et al: It's time for a change to a troponin standard. Circulation 102:1216-1220, 2000

27. Kao JT, Wong IL, Lee JY, et al: Comparison of Abbott AxSYM, Behring Opus Plus, DPC Immulite and Ortho-Clinical Diagnostics Vitros ECi for measurement of cardiac troponin I. Ann Clin Biochem 38:140-146, 2001

28. Van Lente F, Martin A, Ratliff NB, et al: The predictive value of serum enzymes for perioperative myocardial infarction after cardiac operations. An autopsy study. J Thorac Cardiovasc Surg 98:704-710, 1989 